\title{
Nonequilibrium Bethe-Salpeter equation for transient photoabsorption spectroscopy
}

\author{
E. Perfetto, ${ }^{1,2}$ D. Sangalli, ${ }^{3}$ A. Marini, ${ }^{3}$ and G. Stefanucci ${ }^{1,2}$ \\ ${ }^{1}$ Dipartimento di Fisica, Università di Roma Tor Vergata, Via della Ricerca Scientifica 1, 00133 Rome, Italy \\ and European Theoretical Spectroscopy Facility (ETSF) \\ ${ }^{2}$ INFN, Laboratori Nazionali di Frascati, Via E. Fermi 40, 00044 Frascati, Italy \\ ${ }^{3}$ Istituto di Struttura della Materia of the National Research Council, Via Salaria Km 29.3, I-00016 Montelibretti, Italy \\ and European Theoretical Spectroscopy Facility (ETSF)
}

(Received 3 July 2015; revised manuscript received 16 October 2015; published 12 November 2015)

\begin{abstract}
In this work, we propose an accurate first-principles approach to calculate the transient photoabsorption spectrum measured in pump-and-probe experiments. We formulate a condition of adiabaticity and thoroughly analyze the simplifications brought about by the fulfillment of this condition in the nonequilibrium Green's function (NEGF) framework. Starting from the Kadanoff-Baym equations, we derive a nonequilibrium BetheSalpeter equation (BSE) for the response function that can be implemented in most of the already existing ab initio codes. In addition, the adiabatic approximation is benchmarked against full NEGF simulations in simple model Hamiltonians, even under extreme, nonadiabatic conditions in which it is expected to fail. We find that the nonequilibrium BSE is very robust and captures important spectral features in a wide range of experimental configurations.
\end{abstract}

DOI: 10.1103/PhysRevB.92.205304

PACS number(s): 78.47.J-, 31.15.A-, 32.80.Wr

\section{INTRODUCTION}

The impressive progress in ultrafast and ultrastrong laserpulse technology has paved the way to modern nonequilibrium (NEQ) attosecond spectroscopies [1-5]. Unlike conventional spectroscopies, the sample is driven away from equilibrium by a strong laser pulse (the pump) before the photoabsorption of a weaker (perturbative) probe field is measured. Photoabsorption pump-and-probe (PP) spectroscopy experiments are carried out using pump pulses with frequency in the infrared-ultraviolet range and ultrashort probe pulses (down to a few hundred attoseconds). By varying the delay between the pump and probe pulses, one can monitor the excited-state dynamics in a wide energy range.

For samples of linear dimension (in the case of extended systems, this is the dimension of the primitive cell) smaller than the wavelength of the incident light, the measured signal can be calculated theoretically from the NEQ density response function [6-12] $\chi$ (dipole approximation) or, equivalently, from equilibrium dipole correlators of order larger than 2 [13-18]. In the present paper, we follow the first path.

At equilibrium, $\chi(\omega)$ can be used to construct the dipoledipole correlation function $\alpha(\omega)$ in an isolated system or the dielectric function $\epsilon(\omega)$ in extended systems. For correlated systems, the calculation of $\chi$ is, in general, a difficult task, and one has to resort to approximations. The most suitable manybody scheme to implement depends on the sample. For atomic or small molecular samples, the configuration interaction (CI) scheme consists in expanding the many-body state in Slater determinants to obtain eigenstates and eigenvalues. Subsequently, the oscillator strengths are computed and used to construct $\chi$ from a Lehmann representation. For molecules with tens of nuclei or more, as well as for crystals, the number of CI configurations is too large for present-day computational capabilities, and alternative (statistical in nature) approaches are required. One such approach is many-body perturbation theory (MBPT). In MBPT, the two-particle electron-hole propagator $L$ satisfies a diagrammatic equation known as the
Bethe-Salpeter equation (BSE), and $\chi$ is constructed from a space-time contraction of the arguments of $L[19,20]$. The BSE has been successfully applied to study photoabsorption spectroscopy of systems ranging from small molecules to bulk metals and insulators. In this context, the BSE is solved at the $G W$ level with a statically screened interaction [19,21-26].

Another convenient alternative to CI (and MBPT) is the linear response (LR) time-dependent density-functional theory (TDDFT). [27,28] Although TDDFT is in principle exact [29,30], the available functionals for actual calculations are based on the adiabatic local density approximation (ALDA) [31-33]. It is well known that ALDA functionals fail in capturing double excitations $[34,35]$, charge transfer excitations [36-38], or the Coulomb blockade phenomenon [39,40] in equilibrium systems. For extended systems, ALDA performs poorly in the description of the response function as it misses the long-range electron-hole interaction needed to describe excitons $[22,41]$. Therefore, the applicability of LR-TDDFT is at present restricted to systems in which the direct electronhole interaction is not too strong, and hence the spectrum is dominated by single particle-hole excitations.

Similarly to the equilibrium case, the PP photoabsorption spectrum is described by the NEQ response function $\chi\left(t, t^{\prime}\right)$. In this work, we identify a set of constraints between characteristic times that allows us to rewrite $\chi\left(t, t^{\prime}\right)$ as a function of the delay $\tau$ between the pump and probe pulses and of the time difference $t-t^{\prime}$, i.e., $\chi\left(t, t^{\prime}\right)=\chi^{\tau}\left(t-t^{\prime}\right)$. Henceforth, we will refer to this approximation as the adiabatic approximation. The mathematical rigorous definition of the adiabatic approximation as well as its testing in a PP setup is the central objective of the present paper.

The adiabatic response function can be computed at different levels of accuracy depending on the theoretical scheme used. In the CI approach, the time-dependent expansion coefficients are used to calculate the time-dependent product of oscillator strengths, and subsequently these products are inserted into a Lehmann-like representation of the NEQ adiabatic $\chi^{\tau}(\omega)$ to yield a PP spectrum with a time-dependent 
modulation of the peak intensity $[8,10,42,43]$. Within MBPT, instead, we show that the equation of motion for $\chi^{\tau}\left(t-t^{\prime}\right)$ can be rewritten as a BSE. The main difference with the equilibrium BSE is that the equilibrium single-particle density matrix is replaced by its time-dependent value as, for instance, obtained from the solution of a Boltzmann-like equation [44]. The NEQ adiabatic $\chi^{\tau}(\omega)$ could also be computed within LR-TDDFT. However, it is reasonable to expect that the performance of ALDA functionals does not improve in NEQ situations.

The structure of the paper is as follows. Section II presents a brief self-contained introduction to the link between the macroscopic observable and the microscopic theory. We discuss both the real-time (Sec. II A) and the response function (Sec. II B) representations. Here we also identify a set of characteristic times in terms of which the condition of adiabaticity is formulated. The MBPT approach to $\chi$ is developed in Sec. III where we introduce the nonequilibrium Green's functions (NEGF) [20,44-48]. The NEGF approach is computationally more expensive than TDDFT, but it has the advantage of including dynamical correlations in a nonperturbative diagrammatic fashion. To reduce the numerical cost, we implement, in Sec. III A, NEGF within the generalized Kadanoff-Baym ansatz [44,49] (GKBA) and then derive the linear response equations in Sec. III B. Except for the GKBA, no other approximations are made at this stage. The complexity of the problem is further reduced in Sec. IV. Here we exploit the adiabatic approximation and obtain the central result of this work, namely a NEQ-BSE. We examine differences and analogies with the more standard equilibrium BSE and discuss the possibility of converting the NEQ-BSE into a Dyson-like equation in Sec. IV A. Finally, in Sec. V we illustrate the theory in a model system by benchmarking the performance of the NEQ-BSE against full NEGF calculations. A summary of the paper and concluding remarks are presented in Sec. VI.

\section{THE TRANSIENT PHOTOABSORPTION SPECTRUM}

In this section, we relate the macroscopic quantity measured in a PP experiment to the microscopic quantum-mechanical properties of the probed sample. This link establishes a connection between the experimental signal and the solution of the complex quantum kinetic equation for the one-particle density matrix.

\section{A. A real-time approach}

In a PP experiment, the transient photoabsorption spectrum of a system driven out of equilibrium by a pump field is measured. The theoretical description of the driven system is achieved by evolving the many-body state in the simultaneous presence of the pump field and of a weak probe field. Let $\mathbf{E}$ and $\mathbf{e}$ be the electric pump and the probe field, respectively. We define the different terms constituting the many-body Hamiltonian $\hat{H}(t)$ according to

$$
\begin{aligned}
\hat{H}^{0} & =\hat{T}+\hat{V}_{N}, \\
\hat{H}^{\mathrm{eq}} & =\hat{H}^{0}+\hat{V}_{e e}, \\
\hat{H}^{\mathrm{neq}}(t) & =\hat{H}^{\mathrm{eq}}+\mathbf{E}(t) \cdot \hat{\mathbf{d}}, \\
\hat{H}(t) & =\hat{H}^{\mathrm{neq}}(t)+\mathbf{e}(t) \cdot \hat{\mathbf{d}} .
\end{aligned}
$$

Here $\hat{T}$ is the kinetic energy operator, $\hat{V}_{N}$ the external static potential of the nuclei, and $\hat{V}_{e e}$ is the electron-electron interaction. Therefore, $\hat{H}^{\mathrm{eq}}$ is the Hamiltonian of the unperturbed system. The inclusion of other interactions, e.g., the electron-phonon interaction, does not modify the derivation and the results of the present section. The terms $\mathbf{E}(t) \cdot \hat{\mathbf{d}}$ and $\mathbf{e}(t) \cdot \hat{\mathbf{d}}$ describe the coupling of the electrons with the pump and probe fields in the dipole approximation, $\hat{\mathbf{d}}$ being the dipole operator (see below for its mathematical definition). For simplicity, we consider linearly polarized pump and probe fields:

$$
\begin{aligned}
\mathbf{E}(t) & =\eta_{P} E(t), \\
\mathbf{e}(t) & =\eta_{p} e(t),
\end{aligned}
$$

with $\eta_{P}$ and $\eta_{p}$ the polarization vectors. The generalization to other kind of polarizations is straightforward.

We work in the second quantization formalism and introduce a suitable single-particle basis with orthonormal wave functions $\left\{\varphi_{i}(\mathbf{r})\right\}$. Then the creation and annihilation field operators $\hat{\psi}^{\dagger}(\mathbf{r})$ and $\hat{\psi}(\mathbf{r})$ for a particle at position $\mathbf{r}$ in space are expanded according to $\hat{\psi}(\mathbf{r})=\sum_{i} \varphi_{i}(\mathbf{r}) \hat{c}_{i}$. The one-particle density-matrix operator takes the form

$$
\hat{\rho}\left(\mathbf{r}, \mathbf{r}^{\prime}\right)=\hat{\psi}^{\dagger}(\mathbf{r}) \hat{\psi}\left(\mathbf{r}^{\prime}\right)=\sum_{i j} \varphi_{i}^{*}(\mathbf{r}) \varphi_{j}\left(\mathbf{r}^{\prime}\right) \hat{\rho}_{j i},
$$

with $\hat{\rho}_{j i}=\hat{c}_{i}^{\dagger} \hat{c}_{j}$. Similarly, the dipole operator projected along the probe field in the $\left\{\varphi_{i}(\mathbf{r})\right\}$ basis reads

$$
\hat{d}=\boldsymbol{\eta}_{p} \cdot \hat{\mathbf{d}} \equiv \int d \mathbf{r}\left(\boldsymbol{\eta}_{p} \cdot \mathbf{r}\right) \hat{\rho}(\mathbf{r}, \mathbf{r})=d_{i j} \hat{\rho}_{j i},
$$

with $d_{i j}=\int d \mathbf{r} \varphi_{i}^{*}(\mathbf{r})\left(\boldsymbol{\eta}_{p} \cdot \mathbf{r}\right) \varphi_{j}(\mathbf{r})$ the dipole matrix elements. In Eq. (4) and in the remainder of the paper, we use the Einstein convention that repeated indices are summed over. The timedependent expectation value of the dipole operator is given by

$$
d(t)=\langle\Psi(t)|\hat{d}| \Psi(t)\rangle=d_{i j}\left\langle\Psi(t)\left|\hat{\rho}_{j i}\right| \Psi(t)\right\rangle,
$$

where $|\Psi(t)\rangle$ is the state of the system at time $t$.

Without any loss of generality, we assume that the switch-on time of the pump and probe fields is larger than zero; hence the system is in the ground state $\left|\Psi_{g}\right\rangle$ at time $t=0$. Let $\hat{\mathcal{U}}_{H}(t)$ be the unitary evolution operator corresponding to a system with dynamics $\hat{H}$,

$$
\hat{\mathcal{U}}_{H}(t) \equiv T e^{-i \int_{0}^{t} d \bar{t}[\hat{H}(\bar{t})]}, \quad t>0 .
$$

The time-dependent matrix elements of the one-particle density matrix in the presence of both the pump and probe fields are therefore given by

$$
\rho_{j i}(t) \equiv\left\langle\Psi(t)\left|\hat{\rho}_{j i}\right| \Psi(t)\right\rangle=\left\langle\Psi_{g}\left|\hat{\mathcal{U}}_{H}^{\dagger}(t) \hat{\rho}_{j i} \hat{\mathcal{U}}_{H}(t)\right| \Psi_{g}\right\rangle .
$$

Replacing $\hat{H}$ with $\hat{H}^{\text {neq }}$ in Eq. (6), we have the evolution operator in the presence of the pump only, $\hat{\mathcal{U}}_{H^{\text {neq }}}(t)$. To simplify the notation, we put a tilde on time-dependent expectation values obtained with a probe-free propagation. Thus

$$
\tilde{\rho}_{j i}(t)=\left\langle\Psi_{g}\left|\hat{\mathcal{U}}_{H^{\text {neq }}}^{\dagger}(t) \hat{\rho}_{j i} \hat{\mathcal{U}}_{H^{\text {neq }}}(t)\right| \Psi_{g}\right\rangle,
$$

and hence $\tilde{d}(t)=d_{i j} \tilde{\rho}_{j i}(t)$. 
For optically thin samples [50], the transmitted probe field $\mathbf{e}^{\prime}(t)=\eta_{p} e^{\prime}(t)$ is related to the probe-induced variation $\mathbf{d}_{p}(t) \equiv \mathbf{d}(t)-\tilde{\mathbf{d}}(t)$ of the dipole moment by [12]

$$
\mathbf{e}^{\prime}(t)=\mathbf{e}(t)+\frac{2 \pi}{S c} \frac{d}{d t} \mathbf{d}_{p}(t)
$$

where $S$ is the cross section of the sample (assumed to be smaller than the cross section of the laser beam).

The transmitted probe field is typically split in two halves and then merged back by a spectrometer, thus generating an electric field $\frac{1}{2}\left[\mathbf{e}^{\prime}(t)+\mathbf{e}^{\prime}(t-\delta)\right]$ with a tunable delay $\delta \geqslant 0$. In a PP experiment, the integrated intensity of this field, i.e., the total absorbed energy per unit area, is measured as a function of $\delta$ :

$$
I^{\prime}(\delta)=\frac{c}{4 \pi} \int_{-\infty}^{\infty} d t\left|\frac{\mathbf{e}^{\prime}(t)+\mathbf{e}^{\prime}(t-\delta)}{2}\right|^{2} .
$$

The resulting function $I^{\prime}(\delta)$ is then cosine-transformed,

$$
\Im^{\prime}(\omega)=\int_{0}^{\infty} d \delta I^{\prime}(\delta) \cos (\omega \delta),
$$

to gain information about the absorption energies of the system. Although the probe pulse has a finite duration, the time integral in Eq. (10) goes from minus to plus infinity since the cosine transform requires $I^{\prime}(\delta)$ for all delays $\delta \geqslant 0$.

Performing an analogous spectral decomposition of $\mathbf{e}(t)$, we get the intensity $\mathfrak{I}(\omega)$ of the incident probe field. The photoabsorption spectrum $\mathfrak{S}(\omega)$ is therefore given by the difference

$$
\frac{\mathfrak{S}(\omega)}{S}=\mathfrak{I}(\omega)-\mathfrak{I}^{\prime}(\omega) .
$$

Using Eq. (9), it is straightforward to show that [12]

$$
\mathfrak{S}(\omega)=-2 \omega \operatorname{Im}\left[e(\omega) d_{p}(\omega)\right]-\frac{2 \pi}{S c}\left|\omega d_{p}(\omega)\right|^{2},
$$

where $e(\omega)$ and $d_{p}(\omega)$ are the Fourier transform of the timedependent probe field $e(t)$ and probe-induced dipole moment $d_{p}(t) \equiv d(t)-\tilde{d}(t)$, respectively. This relation expresses the aforementioned link between the macroscopic intensity of the transmitted probe field measured in a PP experiment and the microscopic dipole moment.

In photoabsorption experiments of equilibrium systems (no pump), the induced electric field [second term on the righthand side of Eq. (9)] is typically much smaller than the incident probe, and the quadratic term in the dipole moment appearing in $\mathfrak{S}(\omega)$ can be safely discarded. Moreover, $d_{p}(\omega)=\alpha(\omega) e(\omega)$ with $\alpha(\omega) \equiv d_{i j} \chi_{j i}(\omega) d_{l k}$ having the property that $\operatorname{Im}[\alpha(\omega)] \lessgtr$ 0 for $\omega \gtrless 0$ (see the next section), and therefore the ratio $\mathfrak{S}(\omega) /|e(\omega)|^{2}$ is positive and independent of the shape of the probe. On the contrary, the photoabsorption spectrum of a pump-driven system is not an intrinsic property of the sample since $d_{p}(\omega)$, although still linear in $e$, depends on $e\left(\omega^{\prime}\right)$ at all possible frequencies $\omega^{\prime}$. Translating this statement from frequencies to times, the spectrum depends on the shape of the probe and on the NEQ state of the system at the time the probe pulse enters the sample (hence on the delay between the pump and probe pulses). Furthermore, there might be frequencies for which the spectrum $\mathfrak{S}(\omega)$ is negative due to a dominance of the stimulated emission over absorption.

For a fixed shape of the pump and probe pulse, the main interest in PP experiments is to study the evolution of the spectrum as the delay $\tau$ between the two pulses is varied. Assuming that the quadratic term in $d_{p}$, see Eq. (13), is small and taking into account that $d_{p}(\omega)=d_{i j}\left[\rho_{j i}(\omega)-\tilde{\rho}_{j i}(\omega)\right]$, the resulting spectrum reads

$$
\mathfrak{S}^{\tau}(\omega)=-2 \omega \operatorname{Im}\left[e^{*}(\omega) d_{i j} \delta \rho_{j i}(\omega)\right],
$$

where we define $\delta \rho=\rho-\tilde{\rho}$. In Eq. (14), we explicitly added to $\mathfrak{S}$ a dependence on $\tau$ since the probe field as well as the timedependent density matrix depends on the pump-probe delay. This dependence is, in general, rather complex and difficult to interpret. As we shall see, the calculation of the spectrum as well as its physical interpretation are greatly simplified if the adiabatic approximation is made.

\section{B. A response-function representation: The adiabatic condition}

Equation (14) can be rewritten in a different way using linear response theory out of equilibrium. Let us introduce the (retarded) NEQ response function

$$
\chi_{j k}\left(t, t^{\prime}\right)=-i \theta\left(t-t^{\prime}\right)\left\langle\Psi_{g}\left|\left[\hat{c}_{i H}^{\dagger}(t) \hat{c}_{j H}(t), \hat{c}_{k H}^{\dagger}\left(t^{\prime}\right) \hat{c}_{l H}\left(t^{\prime}\right)\right]\right| \Psi_{g}\right\rangle,
$$

where $\hat{c}_{i H}(t) \equiv \hat{\mathcal{U}}_{H^{\text {neq }}}^{\dagger}(t) \hat{c}_{i} \hat{\mathcal{U}}_{H^{\text {neq }}}(t)$ are fermion operators in the Heisenberg picture with respect to the probe-free Hamiltonian $\hat{H}^{\text {neq }}$. To first order in $\mathbf{e}$, the probe-induced variation of the dipole moment reads

$$
\begin{aligned}
d_{p}(t) & =d_{i j} \int d t^{\prime} \chi_{j i}\left(t, t^{\prime}\right) d_{k l} e\left(t^{\prime}\right) \\
& =\int d t^{\prime}\left[d \circ \chi\left(t, t^{\prime}\right) \circ d\right] e\left(t^{\prime}\right) .
\end{aligned}
$$

In Eq. (16), we introduced a short-hand notation for the contraction of tensors of different rank. Below we define the four types of contractions, which include the one in Eq. (16), that we use in the paper:

$$
\begin{aligned}
& (M \circ V)_{p q} \equiv \underset{m n}{M_{p q} V_{n m},} \\
& (T \circ M \circ V) \equiv T_{p q} M_{q p} V_{n m}, \\
& (M \circ N)_{m n} \equiv M_{m n} N_{q p}, \\
& {[N, V]_{m n}=-[V, N]_{p q} \equiv \underset{p q}{N_{m i}} V_{i n}-V_{m i} N_{i n} .}
\end{aligned}
$$

The rank of the tensors will be clear from the context. Notice that Eq. (17d) has the same structure of a commutator since the lower indices are fixed. Taking into account Eq. (14), we clearly see from Eq. (16) the relation between the PP spectrum and the NEQ response function; we can also appreciate the complex time dependence introduced by the pump field. In fact, in equilibrium (no pump) the response function reduces to a function of $\left(t-t^{\prime}\right)$ due to the invariance under time 
translations. Using this invariance, the linear response relation Eq. (16) in Fourier space reads $d_{p}(\omega)=\alpha(\omega) e(\omega)$ with $\alpha=$ $(d \circ \chi \circ d)$, and the ratio $\mathfrak{S}(\omega) /|e(\omega)|^{2}$ becomes independent of the probe. As already discussed in the introduction, the equilibrium response function can be calculated by solving the BSE.

In the time domain, the equation for the electron-hole propagator $L$ ( $\chi$ follows from a space-time contraction of $L)$ is valid out-of-equilibrium too. The most convenient and compact way to write the nonequilibrium equation for $L$ is through the many-particle Green's functions on the Keldysh contour [20]. Let us introduce the short-hand notation $1=\left(i_{1}, z_{1}\right), 2=\left(i_{2}, z_{2}\right)$, etc. for the orbital and contour-time coordinates. Then, the one- and two-particle Keldysh Green's functions are defined according to

$$
\begin{gathered}
G(1 ; 2) \equiv \frac{1}{i}\left\langle\mathcal{T}\left\{\hat{c}_{i_{1} H}\left(z_{1}\right) \hat{c}_{i_{2} H}^{\dagger}\left(z_{2}\right)\right\}\right\rangle \\
G_{2}(1,2 ; 3,4) \equiv \frac{1}{i}\left\langle\mathcal{T}\left\{\hat{c}_{i_{1} H}\left(z_{1}\right) \hat{c}_{i_{2} H}\left(z_{2}\right) \hat{c}_{i_{4} H}^{\dagger}\left(z_{4}\right) \hat{c}_{i_{3} H}^{\dagger}\left(z_{3}\right)\right\}\right\rangle
\end{gathered}
$$

where $\mathcal{T}$ is the contour ordering operator and the average is over the ground state $\Psi_{g}$. The Keldysh electron-hole propagator $L$ then reads

$$
L(1,2 ; 3,4)=-G_{2}(1,2 ; 3,4)+G(1 ; 3) G(2 ; 4),
$$

and it satisfies the four-point equation

$$
\begin{aligned}
L(1,2 ; 3,4)= & G(1 ; 4) G(2 ; 3)-\int G\left(1 ; 1^{\prime}\right) G\left(3^{\prime} ; 3\right) \\
& \times K\left(1^{\prime}, 2^{\prime} ; 3^{\prime}, 4^{\prime}\right) L\left(4^{\prime}, 2 ; 2^{\prime}, 4\right)
\end{aligned}
$$

with the four-point kernel

$$
K(1,2 ; 3,4)=\delta \Sigma(1 ; 3) / \delta G(4 ; 2),
$$

given by the functional derivative of the self-energy with respect to the Green's function. In Eq. (21), the integral is over all primed variables and it implies a sum over orbital indices and an integral over the contour times. If we could solve Eq. (21) for $L$, then the Keldysh response function would follow from [20],

$$
\underset{l k}{\chi_{j i}}\left(z_{1}, z_{2}\right)=-i L\left(j z_{1}, l z_{2} ; k z_{2}^{+}, i z_{1}^{+}\right)
$$

where $z^{+}$indicates a contour time infinitesimally later than $z$. The NEQ (retarded) response function in Eq. (15) would then read

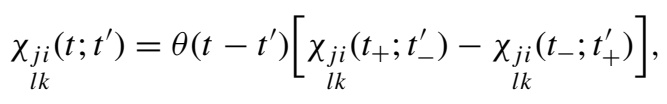

where $z=t_{-}\left(z=t_{+}\right)$is the contour time on the backward (forward) branch of the Keldysh contour.

The numerical solution of Eq. (21) is essentially impossible for present-day computational capabilities. The problem is therefore to find a simple but still accurate approach to calculate the NEQ $\chi$ within MBPT. For this purpose, we will extend the equilibrium BSE to NEQ situations relevant to PP experiments and provide a sound interpretation of the two-time dependence. In the following, we refer to this equation as the NEQ-BSE.

We begin the discussion by introducing two fundamental characteristic times that support the adiabatic approximation: the key idea is that a NEQ-BSE is meaningful whenever the system is substantially frozen in a NEQ configuration during the measurement process. The characteristic times are as follows:

(i) The time scale $T_{P}$ of the electron dynamics induced by the pump. If $\Delta t \ll T_{P}$, then $\tilde{\rho}(t+\Delta t) \approx \tilde{\rho}(t)$.

(ii) The lifetime $\tau_{p}$ of the dressed probe pulse, which is the duration of the measurement process too.

We can formulate the condition of applicability of the adiabatic approximation as

$$
T_{P} \gg \tau_{p}
$$

Equation (25) expresses the physical condition that the probefree $\tilde{\rho}(t)$ has to vary on a time scale $\left(T_{P}\right)$ much longer than the duration $\left(\tau_{p}\right)$ of the dressed probe. Of course for $\tau_{p}$ to be smaller than typical electronic time scales, there should exist decay channels faster than the radiative decay. This is the case of solid slabs as well as of thick atomic or molecular gases. The following analysis applies to this class of systems.

We identify two different situations in which the condition in Eq. (25) is fulfilled. (i) If the pump itself varies on a time scale $T_{P} \gg \tau_{p}$, then Eq. (25) is always fulfilled since the pump-induced dynamics cannot be faster than $T_{P}$. In this case, the adiabatic approximation, and hence the NEQ-BSE, can be used to describe the transient spectrum for any delay $\tau$ between the pump and probe fields. (ii) In general, however, the pump is a pulse of duration $\Delta_{P}$ (see Fig. 1), no longer than a few hundred femtoseconds capable of inducing arbitrary fast processes. During the action of the pump, the level occupations change and the system polarizes. Shortly after $\Delta_{P}$, we have a transient period characterized by a dephasing-driven drop of the pump-induced polarization, we denote by $\tau_{\text {pol }}$ the polarization lifetime in this nonequilibrium situation, and by a stabilization of the level occupations at some nonequilibrium value, we denote by $\tau_{\text {carr }}$ the characteristic time for the occupations to stabilize; see again Fig. 1. Thus, after a time $\tau_{\max }=\max \left(\tau_{\text {pol }}, \tau_{\text {carr }}\right)$, typically $\tau_{\text {pol }}<\tau_{\text {carr }}$, we may say that the system is in a quasistationary state with carriers in some excited levels. In this quasistationary regime, the time to relax back to the ground state is dictated by scattering processes (electron-electron, electron-phonon, and electron-photon) and can be of the order of picoseconds. If we denote by $\tau_{\text {scatt }}$ this relaxation time scale, then we have $T_{P}=\tau_{\text {scatt }}$. Suppose now to probe the system in this quasistationary state with a pulse $e(t)$ of duration $\Delta_{p}$. The probe induces a polarization $d_{p}(t)$ that dresses the bare $e(t)$ and, in general, has a finite lifetime $\tilde{\tau}_{p}$. Hence the duration of the dressed probe field, which coincides with the duration of the measurement process, is $\tau_{p}=\Delta_{p}+\tilde{\tau}_{p}$. In this regime, the condition in Eq. (25) is fulfilled provided that $\tau_{p}$ is shorter than the relaxation time $\tau_{\text {scatt }}$. This is often the case as $\tau_{p}$ is typically in the femtosecond range.

In Fig. 1, we represent the dressed probe field with oscillations of frequency $\omega_{p}$. Although the characteristic 


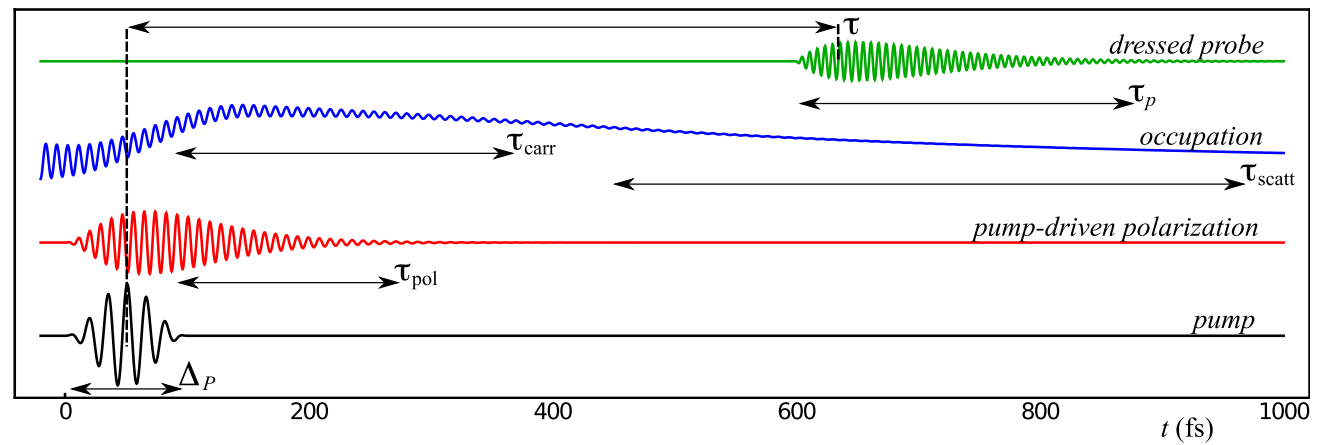

FIG. 1. (Color online) Illustration of the characteristic times described in the main text: $T_{P}$ is the time scale of the electron dynamics induced by the pump, $\tau_{\mathrm{pol}}$ is the dephasing time of the pump-induced polarization, $\tau_{\text {carr }}$ is the stabilization time of the occupations, $\tau_{\text {scatt }}$ is the time to relax back to the equilibrium state, and $\tau_{p}$ is the lifetime of the dressed probe field. We also display the delay $\tau$ between pump and probe.

frequency $\omega_{p}$ can be any, it is clear that it is only for

$$
\tau_{p} \gg \frac{2 \pi}{\omega_{p}},
$$

that the Fourier transform of the probe-induced dipole has a well-defined structure in $\omega_{p}$. This implies that the lifetime $\tau_{p}$ also sets a lower limit to the frequency resolution of a transient spectroscopy experiment.

When the inequality of Eq. (25) is satisfied, the probe sees a NEQ frozen system. If we take $t=0$ as the time at which the pump is on, then the probe acts at $t=\tau$ and for times $\left(t, t^{\prime}\right) \in\left[\tau-\tau_{p}, \tau+\tau_{p}\right]$ the response function

$$
\chi\left(t, t^{\prime}\right) \approx \chi^{\tau}\left(t-t^{\prime}\right)
$$

depends only on $\left(t-t^{\prime}\right)$ to a large extent. We emphasize that in Eq. (27) the delay $\tau$ defines the boundary of the $\left(t, t^{\prime}\right)$ domain and it is not the center-of-mass time or something similar. Therefore, Eq. (27) represents a nontrivial statement since it is not possible to reconstruct $\left(t, t^{\prime}\right)$ from $\tau$ and $t-t^{\prime}$. We will provide a more precise definition of $\chi^{\tau}\left(t-t^{\prime}\right)$ in the next section. For the time being, we observe that whenever we can make the adiabatic approximation of Eq. (27), the transient photoabsorption spectrum of Eq. (14) can be written as

$$
\mathfrak{S}^{\tau}(\omega)=-2 \omega|e(\omega)|^{2} \operatorname{Im}\left[d \circ \chi^{\tau}(\omega) \circ d\right] .
$$

Consequently, the ratio $\mathfrak{S}^{\tau}(\omega) /|e(\omega)|^{2}$ becomes independent of the probe and can be interpreted as an intrinsic property of the nonequilibrium system; see again the discussion below Eq. (13).

As a very general remark, we notice that when the system is probed after the pump (no overlap between the pulses), the probe-induced dipole moment oscillates at frequencies $\Omega_{\alpha \beta}=$ $E_{\alpha}-E_{\beta}$, where $E_{\alpha}, E_{\beta}$ are eigenenergies of $\hat{H}^{\text {eq }}$; see Eqs. (15) and (16) $[12,51]$. Furthermore, the amplitude of the oscillations depends on the delay $\tau$ [12]. Therefore, PP spectra are richer than equilibrium spectra where the probe-induced dipole moment can oscillate only at frequencies $\Omega_{\beta}=E_{g}-E_{\beta}$, with $E_{g}$ the ground-state energy, with constant amplitudes. The extra transitions are usually referred to as photoinduced absorption and stimulated emission.

To summarize, Eqs. (14) and (28) represent two different ways of calculating the transient photoabsorption spectrum.
We could either perform a time propagation with both the pump and the probe, a second time propagation with only the pump and then extract the probe-induced dipole moment $\delta d$, or we can evaluate the response function $\chi^{\tau}$ from a NEQ-BSE. The latter approach is developed in the next section.

\section{A NONEQUILIBRIUM GREEN'S-FUNCTION APPROACH TO TRANSIENT ABSORPTION}

In the preceding section, we have introduced the theoretical description of transient absorption experiments with two possible approaches. The first, which is exact, is based on Eq. (14) and the second, which uses the adiabatic approximation, is based on Eq. (28). However, these equations assume that it is ideally possible to compute the exact time-dependent density matrix or the exact adiabatic response function. This is not doable in practice and one has to resort to approximations. In the following, we show how to use NEGF theory to obtain a MBPT equation for $d_{p}(t)$. In the next section, we use this result to generate an equation for the NEQ response function $\chi\left(t, t^{\prime}\right)$, and subsequently we make the adiabatic approximation to derive the NEQ-BSE for $\chi^{\tau}\left(t-t^{\prime}\right)$.

In the MBPT approach, the description in terms of the many-body Hamiltonian containing the electron-electron interaction is replaced by a description in terms of the one-particle Hamiltonian and the many-body self-energy. For simplicity in this work we consider nonmagnetic (spincompensated) systems; the extension to magnetic (or even superconducting) systems is straightforward. We thus define

$$
\begin{aligned}
h^{0} & =\left[-\frac{\nabla^{2}}{2}+V_{N}(\mathbf{r})\right], \\
h^{\mathrm{eq}} & =h^{0}+\Sigma^{0}, \\
\tilde{h}^{t}(t) & =h^{\mathrm{eq}}+\Delta \tilde{\Sigma}_{s}^{t}+\mathbf{E}(t) \cdot \mathbf{d}, \\
h^{t}(t) & =\tilde{h}^{t}(t)+\delta \Sigma_{s}^{t}+\mathbf{e}(t) \cdot \mathbf{d} .
\end{aligned}
$$

We have introduced here the $t$ superscript to indicate a quantity whose time dependence is given by the implicit dependence on the instantaneous density matrix. This means that for a time-dependent functional $f[\rho(t)]\left(t^{\prime}\right)$ or for a scalar $f[\rho(t)]$ depending exclusively on the density matrix at time $t$, 
we have

$$
\begin{aligned}
f^{t} & \equiv f[\rho(t)], \\
f^{t}\left(t^{\prime}\right) & \equiv f[\rho(t)]\left(t^{\prime}\right) .
\end{aligned}
$$

Furthermore, to indicate that the function is calculated at the probe-free density matrix $\tilde{\rho}$, we put a tilde symbol on the function. Thus $\tilde{f}^{t} \equiv f[\tilde{\rho}(t)]$ and $\tilde{f}^{t}\left(t^{\prime}\right) \equiv f[\tilde{\rho}(t)]\left(t^{\prime}\right)$. Let us define the three different self-energies appearing in Eqs. (29). The self-energy $\Sigma^{0}=\Sigma_{s}\left[\rho^{\mathrm{eq}}\right]$ is the static part of the equilibrium many-body self-energy and it is therefore calculated at the equilibrium density matrix $\rho^{\text {eq }}$. The selfenergies $\Delta \tilde{\Sigma}_{s}^{t}$ and $\delta \Sigma_{s}^{t}$ are the variations due to a change in $\rho$ induced by the pump and the probe, respectively,

$$
\begin{aligned}
\Delta \tilde{\Sigma}_{s}^{t} & \equiv \Sigma_{s}[\tilde{\rho}(t)]-\Sigma_{s}\left[\rho^{\mathrm{eq}}\right]=\tilde{\Sigma}_{s}^{t}-\Sigma^{0}, \\
\delta \Sigma_{s}^{t} & \equiv \Sigma_{s}[\rho(t)]-\Sigma_{s}[\tilde{\rho}(t)]=\Sigma_{s}^{t}-\tilde{\Sigma}_{s}^{t} .
\end{aligned}
$$

In general, $\Sigma_{s}$ is the Hartree-Fock (HF) plus static correlation self-energy. It plays a crucial role as it renormalizes the single-particle level energies and introduces correlation effects (such as electron-hole attraction) also in the polarization function. The different possible approximations to $\Sigma_{s}$ reflect the different kind of physics introduced in the dynamics:

(i) A mean-field potential that mimics the correlation effects. An example is DFT where $\Sigma_{s}^{t}$ is local in space and given by the sum of the Hartree and exchange-correlation potential.

(ii) HF self-energy. In this case no correlation is included. The HF self-energy reads $\Sigma_{s}^{t}=V \circ \rho(t)$, with the fourindex tensor $V_{i j}=2 v_{i m n j}-v_{i m j n}$ and $v_{i m n j}$ the two-electron Coulomb integrals.

(iii) Hartree plus a Coulomb hole and screened exchange (COHSEX) self-energy. In this case, correlation is included using a linear response approximation but dynamical effects are neglected. The COHSEX self-energy reads $\Sigma_{s}^{t}=$ $V^{t} \circ \rho+W_{C}^{t}$ with $V_{i j}^{t}=2 v_{i m n j}-v_{i m j n}^{t}$ and $W_{C}^{t}$ the Coulomb hole potential. In $V^{t}$ the screened exchange interaction reads

$$
v^{t}\left(\mathbf{r}, \mathbf{r}^{\prime}\right) \equiv \int d \overline{\mathbf{r}} \epsilon_{\mathrm{RPA}}^{-1}[\rho(t)](\mathbf{r}, \overline{\mathbf{r}}) v\left(\overline{\mathbf{r}}-\mathbf{r}^{\prime}\right) .
$$

In all cases, the static self-energy is a time-local functional of the density matrix.

\section{A. Real-time dynamics (I): The generalized Kadanoff-Baym ansatz}

In NEGF theory, the key quantities are the lesser, $G^{<}\left(t, t^{\prime}\right)$, and greater, $G^{>}\left(t, t^{\prime}\right)$, Green's functions. These functions are defined according to

$$
\begin{aligned}
& G_{i j}^{<}\left(t, t^{\prime}\right)=i\left\langle\hat{c}_{j H}^{\dagger}\left(t^{\prime}\right) \hat{c}_{i H}(t)\right\rangle, \\
& G_{i j}^{>}\left(t, t^{\prime}\right)=-i\left\langle\hat{c}_{i H}(t) \hat{c}_{j H}^{\dagger}\left(t^{\prime}\right)\right\rangle .
\end{aligned}
$$

It is easy to verify that the one-particle density matrix is given by the lesser Green's function at equal times, $\rho(t) \equiv-i G^{<}(t, t)$. The functions $G^{\lessgtr}$ satisfy a set of coupled equations known as the Kadanoff-Baym equations (KBE)
[20,44-48,52,53]. The KBE are integrodifferential equations with a self-energy kernel depending on both $G^{<}$and $G^{>}$. It is possible to collapse the KBE into a single equation for the one-particle density matrix by making the so called generalized Kadanoff-Baym ansatz (GKBA) [49]. The corresponding equation for $\rho$ reads

$$
\frac{d}{d t} \rho(t)+i\left[h^{t}(t), \rho(t)\right]=-I(t),
$$

where $h^{t}(t)$ is defined in Eq. (29).

The collision integral $I(t)=I[\rho](t)$ on the right-hand side of Eq. (35), in contrast with the static self-energies previously discussed, is nonlocal in time (unless specific approximations are made), i.e., it depends on the full history of $\rho$. The functional form is uniquely determined through the GKBA once an approximation for the correlation self-energy, $\Sigma_{c}$, is made. Let us show how to obtain $I$ starting from its exact $\mathrm{KBE}$ expression and then making the GKBA. From the KBE, we have

$$
I(t)=\int d \bar{t}\left[\Sigma_{c}^{<}(t, \bar{t}) G^{(\mathrm{a})}(\bar{t}, t)+\Sigma_{c}^{(\mathrm{r})}(t, \bar{t}) G^{<}(\bar{t}, t)\right]+\text { H.c. },
$$

with $\Sigma_{c}$ a functional of $G^{<}$and $G^{>}$. The functional form of $\Sigma_{c}$ must be consistent with the choice of $\Sigma_{s}$, i.e., $\Sigma_{c}=\Sigma-\Sigma_{s}$ with $\Sigma$ the full many-body self-energy. Retarded/advanced functions carry a superscript (r)/(a) and are defined in terms of the lesser and greater functions according to

$$
\begin{aligned}
X^{(\mathrm{r})}\left(t, t^{\prime}\right) & =\left[X^{(\mathrm{a})}\left(t^{\prime}, t\right)\right]^{\dagger} \\
& =\theta\left(t-t^{\prime}\right)\left[X^{>}\left(t, t^{\prime}\right)-X^{<}\left(t, t^{\prime}\right)\right],
\end{aligned}
$$

where $X$ can be $G, \Sigma_{c}$, or any other two-time correlator. The GKBA is an ansatz for $G^{\lessgtr}$ which turns $\Sigma_{c}$, and hence the collision integral, into a functional of $\rho$ and $G^{(\mathrm{r}) /(\mathrm{a})}$ :

$$
\begin{aligned}
& G^{<}\left(t, t^{\prime}\right)=-G^{(\mathrm{r})}\left(t, t^{\prime}\right) \rho\left(t^{\prime}\right)+\rho(t) G^{(\mathrm{a})}\left(t, t^{\prime}\right), \\
& G^{>}\left(t, t^{\prime}\right)=+G^{(\mathrm{r})}\left(t, t^{\prime}\right) \bar{\rho}\left(t^{\prime}\right)-\bar{\rho}(t) G^{(\mathrm{a})}\left(t, t^{\prime}\right),
\end{aligned}
$$

where $\bar{\rho}=1-\rho$. To transform $I(t)$ into a functional of the density matrix, and hence to close Eq. (35), one needs to express the propagator $G^{(\mathrm{r})}$ in terms of $\rho$. Depending on the system, there exist optimal approximations to the propagator, the most common one being the quasiparticle $(\mathrm{QP})$ propagator

$$
G^{(\mathrm{r})}\left(t, t^{\prime}\right)=-i \theta\left(t-t^{\prime}\right) T e^{-i \int_{t^{\prime}}^{t} d \bar{t} h^{\mathrm{qp}(\bar{t})} .}
$$

For (small) finite systems, the choice $h^{\mathrm{qp}}=h^{\mathrm{eq}}$ (usually $h^{\mathrm{eq}}$ is the HF single-particle Hamiltonian) is a good choice [54]. For extended systems, however, the lack of damping in $h^{\text {eq }}$ prevents the system from relaxing. In these cases, the propagator is typically corrected by adding non-Hermitian terms given by the quasiparticle lifetimes $h^{\mathrm{qp}}=h^{\mathrm{eq}}+i \gamma$ [55-61].

\section{B. Real-time dynamics (II): The linear regime}

If the probe is a weak perturbation, we can work within a linear response approach. Then $\delta \Sigma_{s}^{t}$ is of first order in $\mathbf{e}$ and the collision integral can be expanded as

$$
I(t) \approx \tilde{I}(t)+\delta I(t) .
$$


Inserting Eq. (40) into Eq. (35) and equating terms of the same order in the probe field, we get two equations, one for $\tilde{\rho}$ and another for $\delta \rho$ (omitting the explicit time dependence from the various quantities):

$$
\begin{aligned}
\frac{d}{d t} \tilde{\rho}+i\left[\tilde{h}^{t}, \tilde{\rho}\right] & =-\tilde{I}, \\
\frac{d}{d t} \delta \rho+i\left[\tilde{h}^{t}, \delta \rho\right]+\left[\delta \Sigma_{s}^{t}+\mathbf{e} \cdot \mathbf{d}, \tilde{\rho}\right] & =-\delta I .
\end{aligned}
$$

As we are in the linear response regime, we can rewrite $\delta \Sigma_{s}^{t}$ and $\delta I$ in terms of kernel functions of the probe-free density matrix $\tilde{\rho}$. The notation introduced now proves useful because it highlights the dependence on $\tilde{\rho}$ and $\delta \rho$ :

$$
\begin{aligned}
\delta \Sigma_{s}^{t} & =\tilde{K}_{s}^{t} \circ \delta \rho(t), \\
\delta I(t) & =\int d \bar{t} \tilde{K}_{c}(t, \bar{t}) \circ \delta \rho(\bar{t}) .
\end{aligned}
$$

The static kernel $\tilde{K}_{s}^{t}$ depends only on the instantaneous $\tilde{\rho}(t)$ whereas the correlation kernel $\tilde{K}_{c}$ depends on the full history of the probe-free density matrix. Furthermore, $\tilde{K}_{c}(t, \bar{t})$ vanishes for $\bar{t}>t$ since $I(t)$ depends on $\rho(\bar{t})$ only for $\bar{t}<t$, as it follows directly from Eq. (36) and the GKBA in Eqs. (38). With Eqs. (42), we can rewrite Eq. (41b) as

$$
\begin{aligned}
& \frac{d}{d t} \delta \rho+i\left[\tilde{h}^{t}, \delta \rho\right]+i\left[\tilde{K}_{s}^{t} \circ \delta \rho+\mathbf{e} \cdot \mathbf{d}, \tilde{\rho}\right] \\
& \quad=-\int d \bar{t} \tilde{K}_{c}(t, \bar{t}) \circ \delta \rho(\bar{t}) .
\end{aligned}
$$

Equation (43) is the many-body equation for the calculation of the probe-induced change of the density matrix. It is worth observing that the occurrence of a two-times kernel in Eq. (43) is a direct consequence of the GKBA, which transforms the collision integral into a functional of the one-time quantity $\rho(t)$. Without the GKBA, the correlation self-energy is a functional of the two-times Green's function, and the equation for $\delta \rho$ would contain the four-times kernel appearing in Eq. (21) for the electron-hole propagator $L$.

In the next section, we combine Eq. (43) with the condition of adiabaticity in Eq. (25) to derive a NEQ-BSE.

\section{NONEQUILIBRIUM BETHE-SALPETER EQUATION}

The next step in the derivation of a BSE in the presence of the pump field is to transform Eq. (43) into an equation for the response function. Toward that end, we use the relation

$$
\chi_{l k}\left(t, t^{\prime}\right)=\frac{\delta \rho_{j i}(t)}{\delta u_{k l}\left(t^{\prime}\right)},
$$

with $u_{k l}(t)=\mathbf{e}(t) \cdot \mathbf{d}_{k l}$. Taking the functional derivative of Eq. (43) with respect to $u\left(t^{\prime}\right)$, we find

$$
\begin{gathered}
\frac{d}{d t} \chi\left(t, t^{\prime}\right)+i\left[\tilde{h}^{t}(t), \chi\left(t, t^{\prime}\right)\right]+i\left[\tilde{K}_{s}^{t} \circ \chi\left(t, t^{\prime}\right)+\mathbb{1} \delta\left(t-t^{\prime}\right), \tilde{\rho}(t)\right] \\
=-\int d \bar{t} \tilde{K}_{c}(t, \bar{t}) \circ \chi\left(\bar{t}, t^{\prime}\right)
\end{gathered}
$$

where we introduced the four-index tensor $\mathbb{1}_{j i}=\delta_{j l} \delta_{i k}$. At zero pump, this equation reduces to the equilibrium BSE,

$$
\begin{aligned}
& \frac{d}{d t} \chi^{\mathrm{eq}}\left(t-t^{\prime}\right)+i\left[h^{\mathrm{eq}}, \chi^{\mathrm{eq}}\left(t-t^{\prime}\right)\right] \\
& \quad+i\left[K_{s}^{\mathrm{eq}} \circ \chi^{\mathrm{eq}}\left(t-t^{\prime}\right)+\mathbb{1} \delta\left(t-t^{\prime}\right), \rho^{\mathrm{eq}}\right] \\
& =-\int d \bar{t} K_{c}^{\mathrm{eq}}(t-\bar{t}) \circ \chi^{\mathrm{eq}}\left(\bar{t}-t^{\prime}\right) .
\end{aligned}
$$

The differences between Eqs. (45) and (46) are as follows:

(i) In the equilibrium limit all quantities depend on the relative time coordinate only.

(ii) $\rho^{\text {eq }}$ is time-independent while $\tilde{\rho}(t)$ is time-dependent.

(iii) The static equilibrium Hamiltonian $h^{\text {eq }}$ is replaced by the time-dependent $\tilde{h}^{t}(t)$.

(iv) The kernels $\tilde{K}_{s}^{t}$ and $\tilde{K}_{c}(t, \bar{t})$ are evaluated at the pumpdriven time-dependent density matrix $\tilde{\rho}$ whereas the kernels $K_{s}^{\mathrm{eq}}$ and $K_{c}^{\mathrm{eq}}(t-\bar{t})$ are evaluated at the static equilibrium density matrix $\rho^{\mathrm{eq}}$.

Due to these points, it is not possible to reduce Eq. (45) to an algebraic equation for $\chi\left(t, t^{\prime}\right)$, as is commonly done in stateof-the-art equilibrium calculations after Fourier transforming with respect to the time difference $t-t^{\prime}$. In Eq. (45), $\chi\left(t, t^{\prime}\right)$ is not a function of $t-t^{\prime}$ and, furthermore, the dependence on $t$ appears both implicitly and explicitly in $\tilde{h}^{t}, \tilde{K}_{s}^{t}, \tilde{K}_{c}$, and $\tilde{\rho}$.

Analytical progress can be made provided that the adiabatic condition, see Eq. (25), is fulfilled. We recall that in this approximation the pump-driven density matrix $\tilde{\rho}(t)$ varies slowly over the lifetime $\tau_{p}$ of the dressed probe. Thus for $t \in\left[\tau-\tau_{p}, \tau+\tau_{p}\right]$ we have

$$
\tilde{\rho}(t) \approx \tilde{\rho}(\tau)
$$

In the same time window, $\mathbf{E}(t) \approx \mathbf{E}(\tau)$ and hence Eq. (47) implies that

$$
\begin{gathered}
\tilde{h}^{t}(t) \approx \tilde{h}^{\tau}(\tau), \\
\tilde{K}_{s}^{t} \approx \tilde{K}_{s}^{\tau} .
\end{gathered}
$$

This is a direct consequence of the fact that the functionals $\tilde{h}^{t}(t)$ and $\tilde{K}_{s}^{t}$ are time-local functionals of $\tilde{\rho}(t)$.

Another simplification brought about by the adiabatic condition is that for times $t, t^{\prime} \in\left[\tau-\tau_{p}, \tau+\tau_{p}\right]$ the retarded Green's function, see Eq. (39), can be approximated as

$$
G^{(\mathrm{r})}\left(t, t^{\prime}\right) \approx-i \theta\left(t-t^{\prime}\right) \exp \left[-i \tilde{h}^{\mathrm{qp}}(\tau)\left(t-t^{\prime}\right)\right] .
$$

Therefore, the adiabatic retarded Green's function is invariant under time translations and it is a local functional of the probefree density matrix at time $\tau$. The crucial consequence of this fact is that the correlation kernel too becomes a function of the time difference only and a time-local functional of $\tilde{\rho}(\tau)$ :

$$
\tilde{K}_{c}\left(t, t^{\prime}\right) \simeq \tilde{K}_{c}^{\tau}\left(t-t^{\prime}\right) .
$$

Taking into account Eqs. (47)-(51), we see that the solution of Eq. (45) is a response function $\chi\left(t, t^{\prime}\right) \simeq \chi^{\tau}\left(t-t^{\prime}\right)$ depending on the delay $\tau$ [through its implicit dependence on $\tilde{\rho}(\tau)$ ] and on the time difference $t-t^{\prime}$.

In the adiabatic approximation, Eq. (45) can be conveniently Fourier-transformed to yield an algebraic equation for 
the frequency-dependent response function,

$$
\begin{aligned}
& -i \omega \chi^{\tau}(\omega)+i\left[\tilde{h}^{\tau}(\tau), \chi^{\tau}(\omega)\right]+i\left[\tilde{K}_{s}^{\tau} \circ \chi^{\tau}(\omega)+\mathbb{1}, \tilde{\rho}(\tau)\right] \\
& \quad=-\tilde{K}_{c}^{\tau}(\omega) \circ \chi^{\tau}(\omega) .
\end{aligned}
$$

This is the aforementioned NEQ-BSE and the main result of the present work. We emphasize that $\chi^{\tau}$ is the response function of the finite system. In the case of extended systems, $\chi^{\tau}$ is equivalent to the macroscopic response function obtained from a supercell calculation where the spatial long-range component of the induced Hartree field (corresponding to its $\mathbf{q} \rightarrow \mathbf{0}$ Fourier component) has been removed [22].

The solution of Eq. (52) requires a preliminary calculation of the one-particle density matrix $\tilde{\rho}(t)$. In the next subsection, we show how to rewrite the NEQ-BSE as a Dyson equation for $\chi^{\tau}$. The NEQ Dyson equation is then compared with its equilibrium counterpart to provide an intuitive physical interpretation of the response function.

\section{Reduction to a Dyson equation}

The NEQ-BSE, Eq. (52), can be implemented in most of the $a b$ initio numerical schemes and codes. However, in order to create an even closer connection to standard implementations of the BSE, we further discuss the approximations and conditions under which Eq. (52) turns into a simple Dyson equation.

The crucial aspect is the choice of the reference basis and its link with the adiabatic approximation. Let us first reexamine the equilibrium case. Consider the representation in which $h^{\text {eq }}$ is diagonal, i.e., $h_{i j}^{\text {eq }}=\delta_{i j} \epsilon_{i}^{\text {eq }}$. Then, the equilibrium density matrix is diagonal too and its entries are the occupation factors of the electronic levels: $\rho_{i j}^{\mathrm{eq}}=\delta_{i j} f_{i}^{\mathrm{eq}}$. In this basis, the Fourier transform of the equilibrium BSE, i.e., Eq. (46), reads

$$
\begin{aligned}
& {\left[\omega \mathbb{1}-\Delta \epsilon^{\mathrm{eq}}+i K_{c}^{\mathrm{eq}}(\omega)\right] \circ \chi^{\mathrm{eq}}(\omega)} \\
& \quad=-\Delta f^{\mathrm{eq}} \circ\left[\mathbb{1}+K_{s}^{\mathrm{eq}} \circ \chi^{\mathrm{eq}}(\omega)\right],
\end{aligned}
$$

where

$$
\left(\Delta \epsilon^{\mathrm{eq}}\right)_{i j}=\left(\epsilon_{i}^{\mathrm{eq}}-\epsilon_{j}^{\mathrm{eq}}\right) \mathbb{1}_{i j}
$$

and

$$
\left(\Delta f^{\mathrm{eq}}\right)_{i j}=\left(f_{i}^{\mathrm{eq}}-f_{j}^{\mathrm{eq}}\right) \mathbb{1}_{i j} .
$$

Introducing the response function

$$
\chi_{0}^{\mathrm{eq}}(\omega) \equiv-\left[\omega \mathbb{1}-\Delta \epsilon^{\mathrm{eq}}+i K_{c}^{\mathrm{eq}}(\omega)\right]^{-1} \circ \Delta f^{\mathrm{eq}},
$$

we can rewrite Eq. (53) in the form normally used in firstprinciples calculations,

$$
\chi^{\mathrm{eq}}(\omega)=\chi_{0}^{\mathrm{eq}}(\omega)+\chi_{0}^{\mathrm{eq}}(\omega) \circ K_{s}^{\mathrm{eq}} \circ \chi^{\mathrm{eq}}(\omega) .
$$

The correlation kernel $K_{c}^{\mathrm{eq}}(\omega)$ appearing in $\chi_{0}^{\mathrm{eq}}$ deserves a comment. In most of the applications, $K_{c}^{\mathrm{eq}}$ is usually replaced by a constant, i.e., $K_{c}^{\mathrm{eq}}(\omega) \approx \eta$. More sophisticated approximations with $\left(K_{c}^{\mathrm{eq}}\right)_{i j} \approx \gamma_{i n} \delta_{i j}+\gamma_{m} \delta_{m n}$ have been explored [55]. In this case, the quasiparticle linewidths $\gamma_{i}$ are calculated from equilibrium MBPT. The approximation of a static correlation kernel is based on the observation that dynamical corrections to the screened interaction are partially canceled by the dynamical effects in the quasiparticle corrections; see Refs. [56,62].

Let us now consider the NEQ-BSE, i.e., Eq. (52). As in the equilibrium case, we would like to introduce a $\chi_{0}$ and turn Eq. (52) into a Dyson equation. However, in the NEQ case, neither $\tilde{\rho}(\tau)$ nor $\tilde{h}^{\tau}(\tau)$ is diagonal in the eigenbasis of $h^{\text {eq }}$. Of course we can rotate the equilibrium basis to have $\tilde{h}^{\tau}(\tau)$ diagonal but, in general, $\tilde{\rho}(\tau)$ has off-diagonal entries in this new basis too. Let $O(\tau)$ be the unitary matrix of the transformation from the equilibrium basis to the adiabatic basis in which $\tilde{h}^{\tau}(\tau)$ is diagonal,

$$
\left[O^{\dagger}(\tau) \tilde{h}^{\tau} O(\tau)\right]_{i j}=\delta_{i j} \tilde{\epsilon}_{i}(\tau) .
$$

The NEQ-BSE Eq. (52) in the adiabatic basis reads

$$
\left[\omega \mathbb{1}-\Delta \tilde{\epsilon}(\tau)+i \tilde{K}_{c}^{\tau}(\omega)\right] \circ \chi^{\tau}(\omega)=-\left[\tilde{\rho}(\tau), \tilde{K}_{s}^{\tau} \circ \chi^{\tau}(\omega)+\mathbb{1}\right],
$$

where the four-index tensor $\Delta \tilde{\epsilon}(\tau)$ is defined as in Eq. (54) with $\epsilon_{i}^{\text {eq }} \rightarrow \tilde{\epsilon}_{i}(\tau)$. Next we define the NEQ response function $\chi_{0}^{\tau}$ according to

$$
\chi_{0}^{\tau}(\omega) \equiv-\left[\omega-\Delta \tilde{\epsilon}(\tau)+i \tilde{K}_{c}^{\tau}(\omega)\right]^{-1} \circ[\tilde{\rho}(\tau), \mathbb{1}],
$$

which generalizes Eq. (56) to nondiagonal density matrices. Using the identity

$$
\left[\tilde{\rho}(\tau), \tilde{K}_{s}^{\tau} \circ \chi^{\tau}(\omega)\right]=[\tilde{\rho}(\tau), \mathbb{1}] \circ \tilde{K}_{s}^{\tau} \circ \chi^{\tau}(\omega),
$$

we can rewrite the NEQ-BSE in a Dyson-like form

$$
\chi^{\tau}(\omega)=\chi_{0}^{\tau}(\omega)+\chi_{0}^{\tau}(\omega) \circ \tilde{K}_{s}^{\tau} \circ \chi^{\tau}(\omega) .
$$

The analogy between Eq. (62) and the standard equilibrium BSE becomes more evident if we make some further approximations that are often used in actual implementations:

(i) $h^{\text {eq }}$ constructed from the dynamical $G W$ self-energy. In this way, the equilibrium basis is the quasiparticles basis whose states are renormalized by dynamical effects.

(ii) To recover the equilibrium limit of standard BSE implementations, the term $\delta \Sigma_{s}^{\tau}$ must be the statically screened COHSEX approximation. This has been proved in Ref. [63]. Other choices could be done in principle for the $\Delta \Sigma_{s}^{\tau}$ term, but, for internal consistency, the same approximation should be used.

With these two approximations in mind, we discuss Eq. (52) in the case of a weak pump field. This condition is often realized in PP experiments as it allows us to photoexcite the system without changing too much its electronic and optical properties. Therefore, weak pump fields provide a noninvasive method to monitor the excited states of the equilibrium system.

For weak pump fields, the density of the excited carriers is small. This implies that we can approximate the orthogonal matrix $O_{i j}(\tau) \approx \delta_{i j}$. In other words, the adiabatic basis and the equilibrium basis are essentially the same. The obvious and physically intuitive consequence of this fact is that the diagonal elements of the density matrix are the NEQ occupations $\tilde{\rho}_{i i}(\tau)=\tilde{f}_{i}(\tau)$, whereas the off-diagonal elements describe the polarization of the system. If the photoexcited carrier density is small, the off-diagonal elements can be neglected and Eq. (60) simplifies to

$$
\tilde{\chi}_{0}^{\tau}(\omega) \equiv-\left[\omega-\Delta \tilde{\epsilon}(\tau)+i \tilde{K}_{c}^{\tau}(\omega)\right]^{-1} \circ \Delta \tilde{f}(\tau),
$$


where the four-index tensor $\Delta \tilde{f}(\tau)$ is defined as in Eq. (55) with $f_{i}^{\text {eq }} \rightarrow \tilde{f}_{i}(\tau)$. In addition, the pump-induced renormalization of the single-particle energy levels is

$$
\tilde{\epsilon}_{i}(\tau) \approx \epsilon_{i}^{\mathrm{eq}}+\mathbf{E}(\tau) \cdot \mathbf{d}_{i i}+\Delta \Sigma_{s, i i}^{\tau},
$$

where $\Delta \Sigma_{s}^{\tau}$ is a time-local functional of the occupations only. The $\tau$-dependent renormalization of the energy levels represents the explanation in MBPT language of the wellknown band-gap renormalization effect, i.e., the reduction of the elemental gap induced by pump-excited carriers, measured experimentally in Ref. [64]. A similar phenomenon has also been described with a first-principles approach [65], although in the equilibrium case. A more detailed discussion on the subject will be presented in future work.

Another consequence of the diagonal structure of the density matrix is that the static kernel too becomes a functional of the NEQ occupations only: $\tilde{K}_{s}^{\tau} \approx \tilde{K}_{s}[\tilde{f}(\tau)]$. This dependence can be used to interpret the renormalization of the electron-hole interaction and hence, in systems with bound excitons, the renormalization of the excitonic binding energy.

\section{A NUMERICAL EXAMPLE}

\section{A. Model}

We illustrate the theory developed in the previous sections by calculating the transient photoabsorption spectrum of a four-level model system with two valence states (orbital quantum numbers $\mu=1,2$ ) and two "conduction" or excited states (orbital quantum numbers $\mu=3,4$ ). In second quantization, the equilibrium Hamiltonian reads

$$
\hat{H}^{\mathrm{eq}}=\sum_{\mu \sigma} \epsilon_{\mu} \hat{n}_{\mu \sigma}+\frac{1}{2} \sum_{\substack{\mu \nu \\ \sigma \sigma^{\prime}}} v_{\mu \nu} \hat{c}_{\mu \sigma}^{\dagger} \hat{c}_{\nu \sigma^{\prime}}^{\dagger} \hat{c}_{\nu \sigma^{\prime}} \hat{c}_{\mu \sigma},
$$

with $\hat{n}_{\mu \sigma}=\hat{c}_{\mu \sigma}^{\dagger} \hat{c}_{\mu \sigma}$ the occupation operator of level $\mu$ with spin $\sigma$. The system is driven out of equilibrium by a strong pulse that pumps electrons from the valence states to the conduction states. In accordance with the notation of Eqs. (1), we consider a pump-dipole coupling of the form

$$
\mathbf{E}(t) \cdot \hat{\mathbf{d}}=E(t) \sum_{\substack{\mu=1,2 \\ \nu=3,4}} \sum_{\sigma}\left(d_{\mu \nu} \hat{c}_{\mu \sigma}^{\dagger} \hat{c}_{\nu \sigma}+\text { H.c. }\right),
$$

where $d_{\mu \nu}=\left\langle\varphi_{\mu}\left|\boldsymbol{\eta}_{P} \cdot \hat{\mathbf{r}}\right| \varphi_{\nu}\right\rangle=\boldsymbol{\eta}_{P} \cdot \mathbf{d}_{\mu \nu}$. After a time $\tau$, the excited system is irradiated by a weak ultrafast probe. The probe-dipole coupling is the same as in Eq. (66) except that the field amplitude $E(t)$ is replaced by the amplitude $e(t)$ of the probe pulse. For the numerical simulations, we choose the amplitudes $E(t)$ and $e(t)$ as [10]

$$
E(t)=E_{0} \sin ^{2}\left(\pi \frac{t}{\Delta_{P}}\right) \sin \omega_{P} t
$$

for $0<t<\Delta_{P}$ and zero otherwise, and

$$
e(t)=e_{0} \sin ^{2}\left(\pi \frac{t-\Delta_{P}-\tau}{\Delta_{p}}\right) \sin \omega_{p}\left(t-\Delta_{P}-\tau\right)
$$

for $0<t-\Delta_{P}-\tau<\Delta_{p}$ and zero otherwise.

The equation of motion for the single-particle density matrix is Eq. (35). For $h^{t}(t)$ we take the HF Hamiltonian (see the discussion just before Sec. III A)

$$
\begin{aligned}
h_{\mu \nu}^{t}(t)= & \delta_{\mu \nu}\left[\epsilon_{\mu}+\sum_{\alpha} 2 v_{\mu \alpha} \rho_{\alpha \alpha}(t)\right]-v_{\mu \nu} \rho_{\nu \mu}(t) \\
& +[E(t)+e(t)] d_{\mu \nu} .
\end{aligned}
$$

For the collision integral, we consider a two-step relaxation approximation (in matrix form)

$$
I(t) \approx\left\{\Gamma^{\mathrm{pol}}(t), \rho(t)-\rho^{\mathrm{qs}}\right\}+\left\{\Gamma^{\mathrm{scatt}}(t), \rho(t)-\rho^{\mathrm{eq}}\right\},
$$

where the curly brackets signify an anticommutator. In Eq. (70), the first term accounts for the dephasing of the pump-induced polarization and is responsible for driving the system toward a quasistationary state described by $\rho^{\mathrm{qs}}$. After the dephasing $\Gamma^{\mathrm{pol}}(t) \approx 0$ and the collision integral is dominated by the second term, which describes the relaxation toward the equilibrium state. The damping matrices $\Gamma^{\mathrm{pol}}(t)=\gamma^{\mathrm{pol}}(t) \mathbb{1}$ and $\Gamma^{\mathrm{scatt}}(t)=\gamma^{\text {scatt }}(t) \mathbb{1}$ are proportional to the identity matrix, thus guaranteeing the conservation of the total number of particles $N=2 \operatorname{Tr}[\rho]$. Since there is no pump-induced dephasing in the absence of the pump, $\Gamma^{\mathrm{pol}}$ is proportional to the amplitude of the pump pulse.

The system has filled valence states and empty conduction states at time $t=0$, hence $\rho^{\mathrm{eq}}=\operatorname{diag}\{1,1,0,0\}$. The model parameters as well as the HF equilibrium configuration can be found in Fig. 2. For the dipole matrix, we use

$$
d=d_{0}\left(\begin{array}{llll}
0 & 0 & 1 & 1 \\
0 & 0 & 1 & 1 \\
1 & 1 & 0 & 0 \\
1 & 1 & 0 & 0
\end{array}\right)
$$

The damping functions $\gamma^{\text {scatt }}$ and the two different $\gamma^{\text {pol }}=$ $\gamma_{1}^{\text {pol }}, \gamma_{2}^{\text {pol }}$ that we consider are illustrated in the bottom panel of Fig. 2. In particular, $\gamma^{\text {pol }}$ is responsible for the relaxation toward the quasistationary density matrix $\rho^{\mathrm{qs}}=$ $\operatorname{diag}\{0.9,0.9,0.1,0.1\}$. For the external fields, we study a pump pulse of duration $\Delta_{P}=66 \mathrm{fs}$ and frequency $\omega_{P}=0.6 \mathrm{eV}$, and a probe of duration $\Delta_{p}=20 \mathrm{fs}$ and frequency $\omega_{p}=0.6 \mathrm{eV}$; the amplitudes $E_{0}, e_{0}$, and $d_{0}$ are chosen to yield $E_{0} d_{0}=0.1 \mathrm{eV}$ and $e_{0} d_{0}=0.001 \mathrm{eV}$.

We calculate $\rho(t)$ in the presence of both pump and probe as well as the probe-free $\tilde{\rho}$ and then extract the probe-induced dipole moment $d_{p}(t)=d_{\mu \nu}\left[\rho_{\nu \mu}(t)-\tilde{\rho}_{\nu \mu}(t)\right]$. Successively, we obtain the transient spectrum of Eq. (14) by Fourier transforming the function $d_{p}(t) \times e^{-t / \tilde{\tau}_{p}}$ with $\tilde{\tau}_{p}=80$ fs the lifetime of the probe-induced dipole. In the figures below, the exponential damping is always included in the probe-induced dipole. The probe-free $\tilde{\rho}$ is also used in Eq. (52) to calculate the adiabatic NEQ response function and hence the transient spectrum according to Eq. (28). The quality of the adiabatic approximation is assessed in different physical situations.

\section{B. Results and discussion}

As we discussed in Sec. II B, the NEQ-BSE is applicable provided that the adiabatic condition is met. This is, for instance, the case in experiments on bulk $\mathrm{Si}$ [66], as recently 


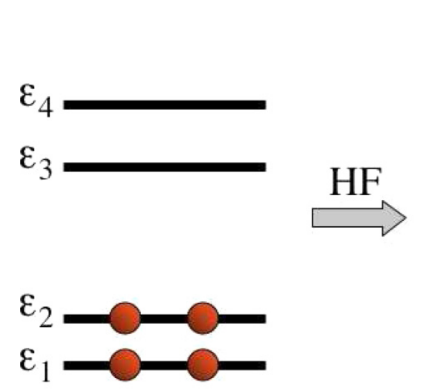

(a)

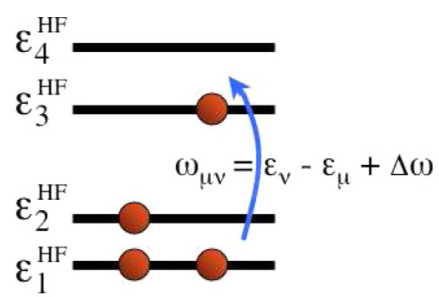

$\Delta \omega=3 v_{13}-2 v_{12}-v_{11}$

(b)

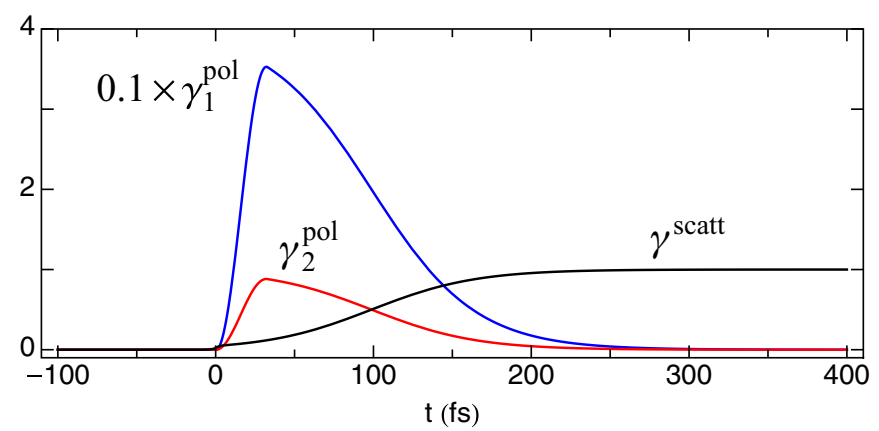

FIG. 2. (Color online) Top panel: (a) noninteracting energy levels $\epsilon_{1}=0, \epsilon_{2}=0.1, \epsilon_{3}=1.0$, and $\epsilon_{4}=1.3$; and (b) HF energy levels $\epsilon_{\mu}^{\mathrm{HF}}=\epsilon_{\mu}+\sum_{\alpha} f_{\alpha}^{\mathrm{eq}}\left(2 v_{\mu \alpha}-\delta_{\mu \alpha} v_{\mu \mu}\right)$ with $v_{\mu \mu}=0.4, v_{12}=v_{23}=$ 0.2 , and otherwise $v_{\mu \nu}=0.1$ (we recall that $f_{\alpha}^{\mathrm{eq}}=\rho_{\alpha \alpha}^{\mathrm{eq}}$ ). The position of the poles $\omega_{\mu \nu}$ of the equilibrium TD-HF $\chi^{\mathrm{eq}}(\omega)$ (solution of Eqs. (56) and (57) with $K_{c}^{\mathrm{eq}}=0$ ) can be calculated analytically and is also indicated. Bottom panel: Plot of the damping functions $\gamma^{\text {scatt }}(t)$, $\gamma_{1}^{\mathrm{pol}}(t)$ (reduced by a factor of 10), and $\gamma_{2}^{\mathrm{pol}}(t)$ used in the numerical simulations. Energies $\epsilon_{\mu}, \epsilon_{\mu}^{\mathrm{HF}}$, and $v_{\mu \nu}$ are in $\mathrm{eV}$ while $\gamma^{\text {scatt }}$ and $\gamma_{1,2}^{\mathrm{pol}}$ are in $\mathrm{meV}$.

shown in Ref. [67]. Here, the pump-induced polarization (off-diagonal elements of the density matrix) damps in a few hundred fs whereas the occupations relax to the initial equilibrium value in a few ps. The damping function $\gamma^{\text {pol }}=$ $\gamma_{1}^{\text {pol }}$ in Fig. 2 (bottom panel) has been devised to reproduce this situation. The four states of the model in Fig. 2 (top panel) can be interpreted as two representative states of the valence band and two representative states of the conduction band. In the top panel of Fig. 3 we show some relevant quantities obtained from the numerical solution of Eq. (35) with $\gamma^{\text {pol }}=\gamma_{1}^{\text {pol }}$, namely (from bottom to top) the pump pulse $E(t)$, the probe-free dipole $\tilde{d}(t)$, the time-dependent occupation $n_{3}(t)=n_{4}(t) \equiv n(t)$ of the valence states 3 and 4 , and the probe-induced dipole $d_{p}(t)$. The behavior of these quantities closely resembles the behavior in Fig. 1 . When the probe arrives ( $\tau=130 \mathrm{fs}$ ), the pump-induced polarization is completely dephased ( $\tau_{\text {pol }} \sim 100 \mathrm{fs}$ ), and the system is slowly moving around the quasistationary excited state described by $\rho^{\mathrm{qs}}$. In this situation, the time scale over which the one-particle density matrix changes is $T_{P} \sim 1 / \gamma^{\text {scatt }} \sim 10^{3}$ fs. Since $T_{P}$ is much larger than the lifetime $\tau_{p}=\tilde{\tau}_{p}+\Delta_{p}=100 \mathrm{fs}$ of the dressed probe, the adiabatic condition is fulfilled; see Eq. (25).

The transient absorption spectra $\mathfrak{S}^{\tau}(\omega)$ obtained within NEGF according to Eq. (14) and with the NEQ-BSE according to Eq. (28) are displayed in Fig. 4. As expected, the NEQ-BSE
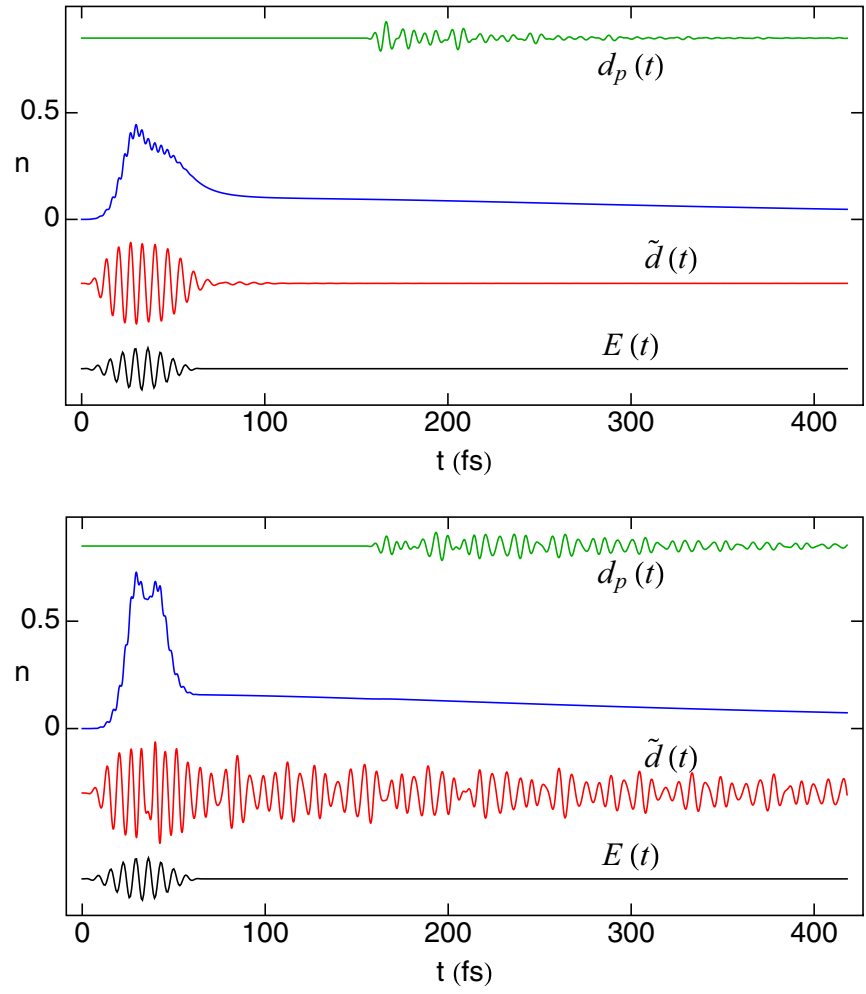

FIG. 3. (Color online) From bottom to top, pump pulse $E(t)$ (black), probe-free dipole $\tilde{d}(t)$ (red), occupation $n_{3}=n_{4} \equiv n$ of valence states (blue), and probe-induced dipole $d_{p}(t)$ (green) for the large $\gamma_{1}^{\text {pol }}$ (top panel) and small $\gamma_{2}^{\text {pol }}$ (bottom panel) damping functions. These results are obtained for a delay $\tau=130 \mathrm{fs}$. The quantities $E(t), \tilde{d}(t)$, and $\delta d(t)$ are in arbitrary units.

approach is very accurate for delays $\tau \lesssim-\tau_{p}$ and $\tau \gtrsim \tau_{\text {pol }}$, i.e., when the probe-induced dipole does not overlap the pump-induced polarization. For $\tau \lesssim-\tau_{p}$, the spectrum $\mathfrak{S}^{\tau}(\omega)$ is the equilibrium spectrum with four peaks at energies $\omega_{\mu \nu}=$ $\epsilon_{v}-\epsilon_{\mu}+\Delta \omega$ (with $\mu=1,2, v=3,4$, and $\Delta \omega=3 v_{13}-$ $\left.2 v_{12}-v_{11}\right)$, thus NEGF and NEQ-BSE obviously agree. For $\tau \gtrsim \tau_{\text {pol }}$, the system is in a nonequilibrium state and the condition of adiabaticity matters. The NEQ-BSE well captures the $\tau$-dependent structure of the NEGF spectrum, with the correct bending of the position of the four main absorption peaks toward their equilibrium value for large $\tau$. At first glance, the agreement seems rather good in the overlapping region $-\tau_{p}<\tau<\tau_{\text {pol }}$ too. However, in this region $\mathfrak{S}^{\tau}(\omega)$ is very small due to the sizable broadening induced by the large $\gamma_{1}^{\text {pol }}$, and a more careful comparison between the NEQ-BSE and NEGF spectra reveals some discrepancies (not shown). Although only two states per band have been considered, the found agreement between NEGF and NEQ-BSE should persist using a more accurate description. In fact, with increasing the number of states the discrete transitions in Fig. 4 do eventually merge to form a continuum but the trend as a function of $\tau$ remains unchanged.

The breakdown of the adiabatic condition is realized in, e.g., finite systems such as atoms or molecules in a strong laser field. For example, in Ref. [42] the level occupations of a gas of excited $\mathrm{Kr}$ ions relax toward the equilibrium values on 

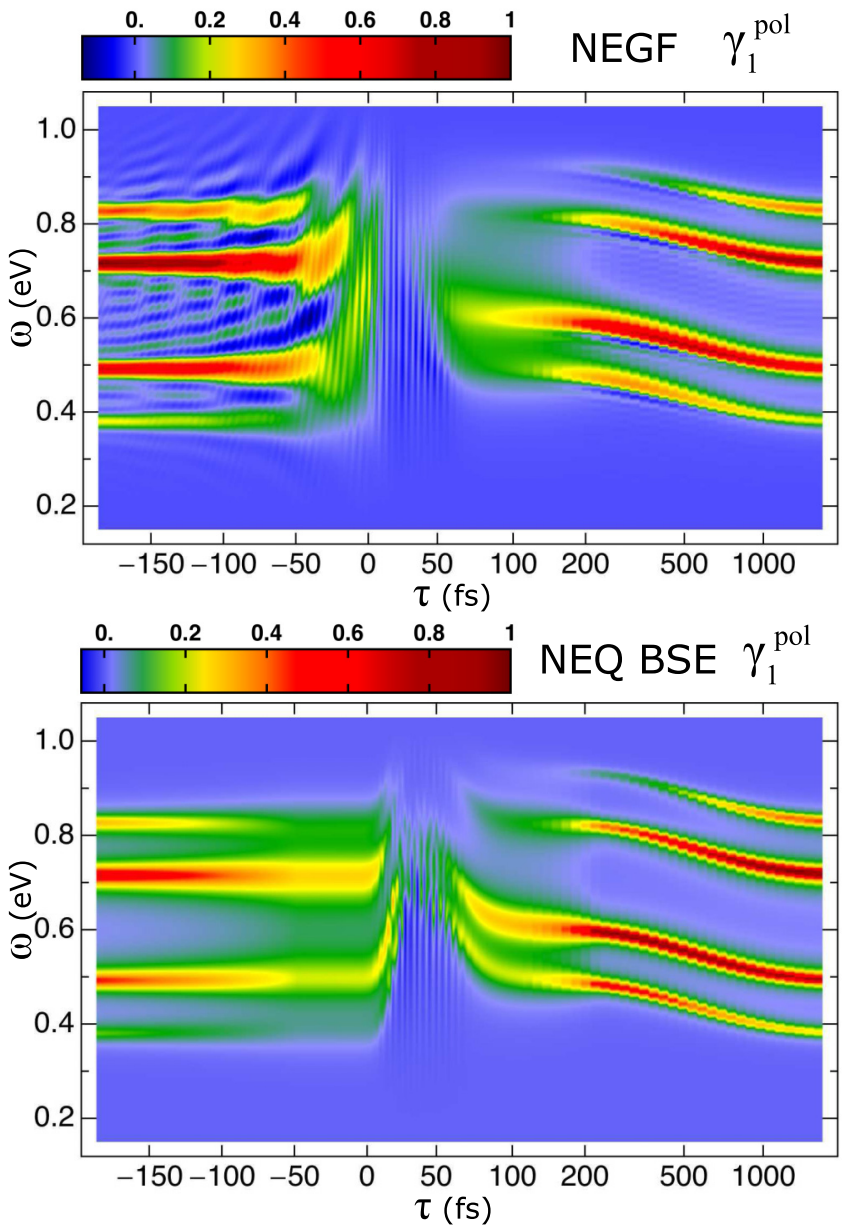

FIG. 4. (Color online) Transient absorption spectrum $\mathfrak{S}^{\tau}(\omega)$ (normalized to its maximum value) obtained within NEGF according to Eq. (14) (top panel) and with the NEQ-BSE according to Eq. (28) (lower panel) using the damping function $\gamma^{\mathrm{pol}}=\gamma_{1}^{\mathrm{pol}}$. The peaks of the NEQ-BSE spectrum have been broadened by the inverse lifetime $1 / \tilde{\tau}_{p}$ of $d_{p}(t)$. Notice that the scale of the horizontal axis is linear for $\tau<100$ fs and logarithmic otherwise.

the same time scale as the pump-induced polarization (the only damping mechanism being the radiative decay). This situation is reproduced by the damping function $\gamma^{\mathrm{pol}}=\gamma_{2}^{\mathrm{pol}}$. As shown in the bottom panel of Fig. 3, the pump-induced polarization $\tilde{d}(t)$ is long-lived. After a time $\tau=130 \mathrm{fs}$, the time scale $T_{P}$ over which the one-particle density matrix changes is given by the period of the coherent oscillations of $\tilde{d}(t)$ and it is roughly equal to the inverse gap $1 / \omega_{23} \approx$ $10 \mathrm{fs}$. Thus the condition of adiabaticity $T_{P} \gg \tau_{p}$ is not fulfilled, and no agreement between NEQ-BSE and NEGF is expected. The transient absorption spectra are displayed in Fig. 5 showing that the two approaches differ whenever the probe experiences a sizable $\tilde{d}(t)$, i.e., for $-\tau_{p}<\tau \lesssim 500$. In this region, the NEGF spectrum exhibits alternating fringes characterized by a large oscillation of the spectral weight at fixed $\omega$ as a function of $\tau$. These features origin from the nonadiabatic coherent motion of the electrons between valence and conduction states, and hence they are out of reach of the NEQ-BSE approach. Remarkably, however, the NEQ-BSE captures important spectral features even in this
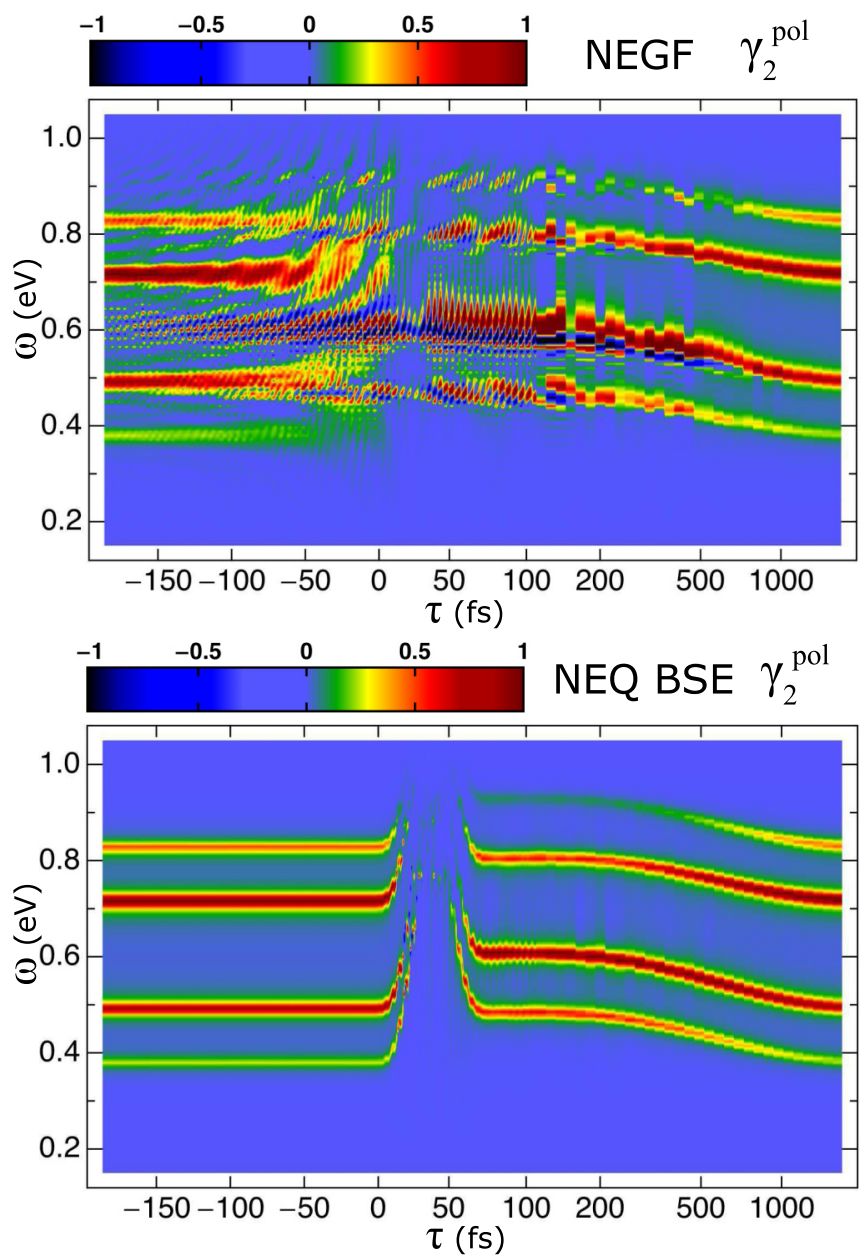

FIG. 5. (Color online) Same as Fig. 4 except that the small damping function $\gamma_{2}^{\mathrm{pol}}$ has been used in Eq. (70).

strongly nonadiabatic situation, the most prominent feature being the upward bending of the main peaks around $\tau=0$. When the coherence is destroyed by the dephasing, i.e., for $\tau>500 \mathrm{fs}$, the NEQ-BSE and NEGF spectra are found to be in excellent agreement.

\section{CONCLUSIONS}

We propose a practical method based on MBPT to calculate PP spectra for delays in the "adiabatic" regime. Starting from the KBE for the Keldysh Green's function, we use the GKBA to obtain an equation of motion for the one-particle density matrix $\rho$ in the presence of both pump and probe fields. Linearization around zero probe yields an equation for the NEQ response function $\chi\left(t, t^{\prime}\right)$. After the action of the pump, we identify a physically relevant regime during which the probe-free density matrix $\tilde{\rho}$ varies on a time scale much longer than the lifetime of the dressed probe. In this regime, we make the adiabatic approximation and show that $\chi\left(t, t^{\prime}\right)$ can be written as a function of the pump-probe delay $\tau$ and of the relative time $\left(t-t^{\prime}\right)$, i.e., $\chi\left(t, t^{\prime}\right) \approx \chi^{\tau}\left(t-t^{\prime}\right)$. This simplification allows us to Fourier transform with respect to the relative time and to derive the main result of this work, namely a NEQ-BSE that can be implemented in most of the ab initio numerical schemes 
and codes. We further provide a sound physical interpretation of the NEQ response function, and we show that it can be related to intrinsic spectral properties of the nonequilibrium system. Well-known effects such as the renormalization of the band gap and excitonic binding energies in semiconductors and insulators are naturally explained.

The computational advantage of the NEQ-BSE over NEGF simulations is enormous as only the probe-free one-particle density matrix $\tilde{\rho}$ enters in the solution of the NEQ-BSE. This implies that a single time propagation is sufficient to obtain the transient spectrum for several delays. In contrast, the NEGF approach requires a time propagation for every delay (to obtain the one-particle density matrix with pump and probe fields) in addition to the time propagation to obtain $\tilde{\rho}$. The validity of the NEQ-BSE has been successfully demonstrated in a simple four-level model system, and it is currently under investigation in more realistic Hamiltonians with encouraging results.

\section{ACKNOWLEDGMENTS}

We acknowledge financial support by the Futuro in Ricerca Grant No. RBFR12SW0J of the Italian Ministry of Education, University and Research MIUR. A.M. and D.S. also acknowledge the funding received from the European Union Horizon 2020 research and innovation program under Grant Agreement No. 654360 ("Nanoscience foundries \& fine analysis" project), and No. 676598 ("Materials design at the eXascale" project).
[1] R. Berera, R. van Grondelle, and J. T. M. Kennis, Photosynth. Res. 101, 105 (2009).

[2] G. Sansone, T. Pfeifer, K. Simeonidis, and A. I. Kuleff, ChemPhysChem 13, 661 (2012).

[3] L. Gallmann, J. Herrmann, R. Locher, M. Sabbar, A. Ludwig, M. Lucchini, and U. Keller, Mol. Phys. 111, 2243 (2013).

[4] A. I. Kuleff and L. S. Cederbaum, J. Phys. B 47, 124002 (2014).

[5] S. M. Falke, C. A. Rozzi, D. Brida, M. Maiuri, M. Amato, E. Sommer, A. De Sio, A. Rubio, G. Cerullo, E. Molinari, and C. Lienau, Science 344, 1001 (2014).

[6] W. Beenken and V. May, J. Opt. Soc. Am. B 14, 2804 (1997).

[7] B. Wolfseder, L. Seidner, G. Stock, and W. Domcke, Chem. Phys. 217, 275 (1997).

[8] R. Santra, V. S. Yakovlev, T. Pfeifer, and Z. Loh, Phys. Rev. A 83, 033405 (2011).

[9] A. S. Moskalenko, Y. Pavlyukh, and J. Berakdar, Phys. Rev. A 86, 013202 (2012).

[10] J. C. Baggesen, E. Lindroth, and L. B. Madsen, Phys. Rev. A 85, 013415 (2012).

[11] A. D. Dutoi, K. Gokhberg, and L. S. Cederbaum, Phys. Rev. A 88, 013419 (2013).

[12] E. Perfetto and G. Stefanucci, Phys. Rev. A 91, 033416 (2015).

[13] Y. Shen, The Principles of Nonlinear Optics, Wiley Series in Pure and Applied Optics (Wiley, New York, 1984).

[14] G. Khitrova, P. R. Berman, and M. Sargent III, J. Opt. Soc. Am. B 5, 160 (1988).

[15] Y. J. Yan and S. Mukamel, Phys. Rev. A 41, 6485 (1990).

[16] W. T. Pollard and R. A. Mathies, Annu. Rev. Phys. Chem. 43, 497 (1992)

[17] S. Mukamel, Principles of Nonlinear Optical Spectroscopy (Oxford University Press, Oxford, 1995).

[18] Y. J. Yan, W. Zhang, and J. Che, J. Chem. Phys. 106, 2212 (1997).

[19] G. Strinati, Riv. Nuovo Cimento 11, 1 (1988).

[20] G. Stefanucci and R. van Leeuwen, Nonequilibrium ManyBody Theory of Quantum Systems: A Modern Introduction (Cambridge University Press, Cambridge, 2013).

[21] G. Strinati, H. J. Mattausch, and W. Hanke, Phys. Rev. B 25, 2867 (1982).

[22] G. Onida, L. Reining, and A. Rubio, Rev. Mod. Phys. 74, 601 (2002).
[23] S. Albrecht, L. Reining, R. Del Sole, and G. Onida, Phys. Rev. Lett. 80, 4510 (1998).

[24] L. X. Benedict, E. L. Shirley, and R. B. Bohn, Phys. Rev. Lett. 80, 4514 (1998).

[25] M. Rohlfing and S. G. Louie, Phys. Rev. Lett. 81, 2312 (1998).

[26] G. Pal, Y. Pavlyukh, W. Hübner, and H. C. Schneider, Eur. Phys. J. B 79, 327 (2011).

[27] M. Petersilka, U. J. Gossmann, and E. K. U. Gross, Phys. Rev. Lett. 76, 1212 (1996).

[28] M. E. Casida, in Recent Advances in Density Functional Methods, Part I, edited by D. P. Chong (World Scientific, Singapore, 1995), p. 155.

[29] E. Runge and E. K. U. Gross, Phys. Rev. Lett. 52, 997 (1984).

[30] C. A. Ullrich, Time-dependent Density-functional Theory: Concepts and Applications (Oxford University Press, Oxford, 2011).

[31] C. A. Rozzi, S. M. Falke, N. Spallanzani, A. Rubio, E. Molinari, D. Brida, M. Maiuri, G. Cerullo, H. Schramm, J. Christoffers, and C. Lienau, Nat. Commun. 4, 1602 (2013).

[32] Ch. Neidel, J. Klei, C.-H. Yang, A. Rouzée, M. J. J. Vrakking, K. Klünder, M. Miranda, C. L. Arnold, T. Fordell, A. L'Huillier, M. Gisselbrecht, P. Johnsson, M. P. Dinh, E. Suraud, P.-G. Reinhard, V. Despré, M. A. L. Marques, and F. Lépine, Phys. Rev. Lett. 111, 033001 (2013).

[33] U. De Giovannini, G. Brunetto, A. Castro, J. Walkenhorst, and A. Rubio, ChemPhysChem 14, 1363 (2013).

[34] N. T. Maitra, F. Zhang, R. J. Cave, and K. Burke, J. Chem. Phys. 120, 5932 (2004).

[35] S. Kümmel and L. Kronik, Rev. Mod. Phys. 80, 3 (2008).

[36] O. Gritsenko and E. J. Baerends, J. Chem. Phys. 121, 655 (2004).

[37] N. T. Maitra, J. Chem. Phys. 122, 234104 (2005).

[38] N. T. Maitra and D. G. Tempel, J. Chem. Phys. 125, 184111 (2006).

[39] G. Stefanucci and S. Kurth, Phys. Rev. Lett. 107, 216401 (2011).

[40] S. Kurth and G. Stefanucci, Phys. Rev. Lett. 111, 030601 (2013).

[41] L. Reining, V. Olevano, A. Rubio, and G. Onida, Phys. Rev. Lett. 88, 066404 (2002).

[42] E. Goulielmakis, Z. Loh, A. Wirth, R. Santra, N. Rohringer, V. S. Yakovlev, S. Zherebtsov, T. Pfeifer, A. M. Azzeer, M. F. Kling, S. R. Leone, and F. Krausz, Nature (London) 466, 739 (2010).

[43] N. Rohringer and R. Santra, Phys. Rev. A 79, 053402 (2009). 
[44] H. Haug and A.-P. Jauho, Quantum Kinetics in Transport and Optics of Semiconductor (Springer-Verlag, Berlin, 1998).

[45] R. van Leeuwen, N. E. Dahlen, G. Stefanucci, C.-O. Almbladh, and U. von Barth, Lect. Notes Phys. 706, 33 (2006).

[46] P. Danielewicz, Ann. Phys. (N.Y.) 152, 239 (1984).

[47] K. Balzer and M. Bonitz, Nonequilibrium Green's Functions Approach to Inhomogeneous Systems, Lecture Notes in Physics Vol. 867 (Springer-Verlag, Berlin, 2013).

[48] L. P. Kadanoff and G. Baym, Quantum Statistical Mechanics (Westview, Boulder, CO, 1994).

[49] P. Lipavský, V. Špička, and B. Velický, Phys. Rev. B 34, 6933 (1986).

[50] Here, by "optically thin" we mean that the sample is microscopic along the propagation direction of the light-pulse.

[51] J. I. Fuks, K. Luo, E. D. Sandoval, and N. T. Maitra, Phys. Rev. Lett. 114, 183002 (2015).

[52] N. E. Dahlen and R. van Leeuwen, Phys. Rev. Lett. 98, 153004 (2007).

[53] P. Myöhänen, A. Stan, G. Stefanucci, and R. van Leeuwen, Phys. Rev. B 80, 115107 (2009).

[54] E. Perfetto, A.-M. Uimonen, R. van Leeuwen, and G. Stefanucci, Phys. Rev. A 92, 033419 (2015).
[55] A. Marini, Phys. Rev. Lett. 101, 106405 (2008).

[56] A. Marini and R. Del Sole, Phys. Rev. Lett. 91, 176402 (2003).

[57] H. Haug, Phys. Status Solidi B 173, 139 (1992).

[58] M. Bonitz, D. Semkat, and H. Haug, Eur. Phys. J. B 9, 309 (1999).

[59] B. Arnaud, S. Lebègue, and M. Alouani, Phys. Rev. B 71, 035308 (2005).

[60] A. Marini, J. Phys.: Conf. Ser. 427, 012003 (2013).

[61] S. Latini, E. Perfetto, A.-M. Uimonen, R. van Leeuwen, and G. Stefanucci, Phys. Rev. B 89, 075306 (2014).

[62] F. Bechstedt, K. Tenelsen, B. Adolph, and R. Del Sole, Phys. Rev. Lett. 78, 1528 (1997).

[63] C. Attaccalite, M. Grüning, and A. Marini, Phys. Rev. B 84, 245110 (2011).

[64] M. Hase, M. Katsuragawa, A. M. Constantinescu, and H. Petek, Nat. Photon. 6, 243 (2012).

[65] A. Schleife, C. Rödl, F. Fuchs, K. Hannewald, and F. Bechstedt, Phys. Rev. Lett. 107, 236405 (2011).

[66] T. Ichibayashi, S. Tanaka, J. Kanasaki, K. Tanimura, and T. Fauster, Phys. Rev. B 84, 235210 (2011).

[67] D. Sangalli and A. Marini, Europhys. Lett. 110, 47004 (2015). 